\title{
¿Por qué hay pocas mujeres científicas? Una revisión de literatura sobre la brecha de género en carreras STEM
}

Why are there few Women Scientists? A Literature Review on the Gender Gap in STEM Careers

\section{Sergio Morales Inga} Bachiller en Antropología, Asistente de investigación, Universidad Nacional Mayor de San Marcos sergio.morales@unmsm.edu.pe

Dr. Oswaldo Morales Tristán ESAN Graduate School of Business omorales@esan.edu.pe
Morales Inga, S. y Morales Tristán, O. (2020) ¿Por qué hay pocas mujeres cientificas? Una revisión de literatura sobre la brecha de género en carreras STEM Revista Internacional de Investigación en Comunicación aDResearch ESIC. № 22 Vol 22

Monográfico especial, marzo 2020 · Págs. 118 a 133 https://doi.org/10.7263/adresic-022-06 
RESUMEN

Clasificación JEL:

J150, J160, 0380

Palabras clave:

Sesgo,

discriminación,

femenino,

equidad de género,

sexismo,

mujer
Alcance de la revisión: El presente ensayo realiza una revisión de literatura sobre la brecha de género en carreras STEM (Science, Technology, Engineering, and Mathematics) y analiza sus principales estudios.

Período de las publicaciones revisadas: Los estudios considerados abarcan desde el año 2005 hasta el año 2019.

Origen y tipos de documentos revisados: La mayoría de la literatura se compone de artículos publicados en revistas indexadas internacionales de sociología, psicología, género y educación.

Aportación del autor sobre las publicaciones analizadas: Los autores proponen una tipificación que organiza los estudios sobre la brecha de género en carreras STEM según los tres tipos de explicación que brindan: a) explicación psicológica, que enfatiza en la instancia del individuo y explica la brecha en términos de autoconcepto, autoconfianza y autoeficacia, creencias y percepciones, y diferencias en intereses y preferencias; b) explicación sociocultural, que enfatiza la importancia de la cultura y explica la brecha por efecto de influencia parental y socialización, discriminación y sesgos, estereotipos y roles de género; y, c) explicación biológica, que enfatiza en los rasgos del sexo anatómico y explica la brecha por su impacto en las diferencias de género.

Conclusiones sobre las líneas de investigación estudiadas: El presente ensayo concluye que la tipificación propuesta sobre los estudios en cuestión permite comprender sus marcos teóricos, instancias centrales de análisis, conceptos eje y principales hallazgos.
ABSTRACT

JEL Classification:

$\mathrm{J} 150, \mathrm{~J} 160,0380$

Key words:

Bias,

discrimination

feminine

gender equity,

sexism,

woman
Reach of the review: This essay conducts a review of the gender gap in STEM careers (Science, Technology, Engineering, and Mathematics) and analyzes its main studies.

Period of the publications reviewed: The studies considered cover from 2005 to 2019

Origin and types of documents reviewed: Most of the literature consists of articles published in international indexed journals of sociology, psychology, gender and education.

Author's contribution on the publications analyzed: The authors propose a typification that organizes studies on the gender gap in STEM careers according to the three types of explanation they provide: a) psychological explanation: which emphasizes the individual's instance and explains the gap in terms of self-concept, self-confidence and self-efficacy, beliefs and perceptions, and differences in interests and preferences; b) sociocultural explanation: which emphasizes the importance of culture and explains the gap due to parental influence and socialization, discrimination and biases, stereotypes and gender roles; and, c) biological explanation: which emphasizes the features of anatomical sex and explains the gap due to its impact on gender differences.

Conclusions on the lines of research studied: This essay concludes that the proposed typification of the studies in question allows us to understand their theoretical frameworks, central analysis instances, core concepts and main findings. 


\section{De la comunicación de la ciencia a la ciencia}

En un reciente estudio, Amarasekara y Grant (2019) analizaron la principal web de distribución de video (YouTube) e indicaron que, de 391 canales de comunicación científica sobre carreras STEM (Science, Technology, Engineering and Mathematics), solo 32 son manejados por mujeres. ¿Por qué la abismal diferencia? Aunque sean minoría, aquellos 32 canales contienen mayores comentarios hostiles, negativos y sexistas. Según los autores, el sexismo y la discriminación, usuales en dicho rubro, evitan que haya más mujeres comunicadoras. ¿Serán esos los únicos motivos? Para comprender dicha problemática cabe empezar por lo básico. La elección de carrera es un área de estudio con amplia historia y teorías diversas (Brown, 2002). Elegir una carrera es un «proceso complejo y multifacético que incluye todas las esferas de la vida de una persona» (Özbilgin y Malach-Pines, 2007, p. 1), es «la elección más crucial en la vida de cualquier estudiante» (Kazi y Akhlaq, 2017, p. 194). Analizar dicho proceso implica comprender los factores que influencian la toma de decisiones: intereses personales, formación profesional, desempeño académico, nivel socioeconómico, historia familiar, demanda laboral o cultura local (Shahzad, Fatima y Ahmed, 2018).

En una revisión de 30 estudios, AkosahTwumasi, Emeto, Lindsay, Tsey y Malau-Aduli (2018) señalaron que la elección de carrera está influenciada por tres grupos de factores: intrínsecos (intereses personales, autoeficacia, expectativas y oportunidades de desarrollo profesional), extrínsecos (salario, estabilidad laboral, prestigio profesional y accesibilidad laboral) e interpersonales (influencia familiar, influencia docente, influencia de pares y responsabilidad social). Rodríguez-Muñiz, Areces, Suárez-Álvarez, Cueli y Muñiz (2019) concluyeron que las motivacio- nes «intrínsecas» (preferencias personales) son más influyentes que las «extrínsecas» (tradición familiar). Asimismo, para Sharif, Ahmed y Sarwar (2019), los padres, la diferenciación social o el salario también influencian la elección de carrera. Como vemos, diversos factores intervienen. ¿Qué diferencias hallamos en los electores respecto su género? ¿Habrá diferencias entre hombres y mujeres en la elección de carrera?

Lightbody, Siann, Tait y Walsh (1997) encuestaron abogadas y médicas, quienes indicaron haber elegido tales carreras por considerar que desempeñan un «rol social útil» al permitir un mayor «involucramiento social». Según los autores, aunque dichas elecciones estén tipificadas por el género (pues hombres y mujeres eligen ocupaciones estereotipadas), «la preferencia femenina por las ocupaciones que involucran 'contacto con las personas', junto con un deseo de estar al servicio de la sociedad en su conjunto, no deben ser infravaloradas» (p. 35). La amplia presencia femenina en carreras humanísticas (frecuentemente investigadas en términos de estereotipos, discriminación, roles sexuales o sexismo) refleja que el género constituye un «factor diferenciador en la elección vocacional y uno de sus condicionantes» (Mosteiro, 1997, p. 308). Asimismo, las creencias sobre el género restringen las aspiraciones profesionales de mujeres.

Según Correll (2004), dichas creencias «pueden evocar estándares diferenciados por género en la atribución del rendimiento a la capacidad, lo que sesga de manera diferencial las evaluaciones que hombres y mujeres hacen de su propia competencia» (p. 94). Incluso en otros contextos geográficos, el género influye al elegir una carrera. En el Sudeste asiático, por ejemplo, hombres y mujeres escogen carreras congruentes con sus roles de género: ellas no eligen profesiones que conflictúen con la vida familiar ni aquellas donde 
la toma de decisiones sea la función principal (Perera y Velummayilum, 2008). Las diferencias de género en percepción de barreras y autoeficacia también son importantes al predecir la elección de carrera (Tien, Wang y Liu, 2009). Asimismo, las creencias religiosas influyen significativamente en las aspiraciones profesionales de las mujeres (Colaner y Giles, 2008).

En el área de la salud, Verde, Gallardo, Compeán, Tamez y Ortiz-Hernández (2007) señalaron que las médicas, enfermeras, nutriólogas y odontólogas eligieron dichas carreras guiadas por estereotipos de género como el prestigio (masculinidad) o la sensibilidad (feminidad). Para los autores, «el género interviene en la definición de ciertos puestos de trabajo, tanto femeninos como masculinos, propiciando la estereotipación sexual de las ocupaciones» (p. 50). Que haya más mujeres en carreras médicas refleja o bien su preferencia por la familia o bien la existencia de barreras en campos dominados por hombres; incluso si hablamos de preferencias ello «ignora hasta qué punto las elecciones profesionales de las mujeres pueden ser decisiones restringidas, influenciadas por factores estructurales que guían a las mujeres en ciertas direcciones y las disuaden de seguir ciertos caminos profesionales» (Kwon, 2017, p. 189). En áreas tecnológicas, el panorama es semejante: la presencia femenina en computación es baja por considerarla un área dominada por hombres (Malik y Al-Emran, 2018).

Dado que las mujeres se han desarrollado profesionalmente en las últimas décadas, ¿qué ocurre con su elección de carrera en términos históricos? Según Ruiz-Gutiérrez y Santana-Vega (2018), el género es importante dado que las mujeres eligen oficios en los que predominan valores como sensibilidad o altruismo, mientras los hombres escogen poder y prestigio. Para los autores, «la mujer históricamente se ha visto atrapada en una sutil tela de araña que se ha estado entretejiendo en torno al mundo femenino: su papel en la sociedad se ha circunscrito al entorno familiar» (p. 16). Analizar cómo el género influye en la elección de carrera es importante para comprender no solo por qué hay pocas comunicadoras científicas, sino también por qué hay pocas científicas; en otras palabras, entender el debate sobre la brecha de género en carreras STEM (en adelante, brecha STEM).

\section{2. ¿Qué es la brecha STEM?}

Diversos estudios dan cuenta de la brecha STEM: hay más hombres que mujeres en carreras científicas, tecnológicas, ingenieriles y matemáticas (Bystydzienski y Bird, 2006; Shapiro y Sax, 2011; Smith, 2011; Sáinz, 2017; Reinking y Martin, 2018). ¿Por qué ocurre ello? La relación mujerciencia es complicada. Desde hace décadas, la subrepresentación femenina en carreras STEM ocurre por motivos diversos: prejuicios, discriminación, estereotipos, sexismo, supuestas incapacidades o estructuras científicas esencialmente masculinas (Fausto-Sterling, 1981). Hoy, tal situación continúa. Según Botella, Rueda, López-Iñesta y Marzal (2019), las mujeres en carreras STEM todavía enfrentan múltiples barreras y retos en distintas etapas de su desarrollo profesional: carencia de tutores, ausencia de modelos femeninos, oportunidades desiguales, sesgos de género y brechas salariales -barreras que muestran impactos diversos y su solución «requeriría la acción de varios actores e instituciones para lograr un cambio real hacia una cultura sensible al género» (p. 13).

Tal brecha se evidencia no solo en la cantidad de científicas, sino también en sus redes de apoyo (Feeney y Bernal, 2010), en diversas formas de acoso (Williams y Massinger, 2016) o en la inscripción a cursos virtuales (Jiang, Schenke, Eccles, Xu y Warschauer, 2018). Es más, para Stoet y Geary (2018), existe una «paradoja de la 
igualdad de género» según la cual las diferencias de género en magnitud de fortalezas académicas relativas y búsqueda de títulos en carreras STEM crecieron con el aumento de la igualdad de género en ciertas regiones; es decir, países con menor equidad de género presentan más mujeres en carreras STEM que los países con mayor equidad. Quizá por ello en América Latina la brecha STEM sea una realidad más urgente que la del mundo anglosajón, dadas sus particularidades históricas (López-Bassols, Grazzi, Guillard y Salazar, 2018; Arredondo, Vázquez y Velázquez, 2019). Considerando su propia complejidad, ¿cómo ha sido explicada la brecha STEM?

\section{Tres explicaciones, un mismo problema}

Aunque los conceptos empleados sean interdependientes, diversos estudios proponen tres tipos de explicación: a) explicación psicológica: enfatiza en la instancia del individuo y explica la brecha en términos de autoconcepto, autoconfianza y autoeficacia, creencias y percepciones, y diferencias en intereses y preferencias; b) explicación sociocultural: enfatiza la importancia de la cultura y explica la brecha por efecto de influencia parental y socialización, discriminación y sesgos, estereotipos y roles de género; y, c) explicación biológica: enfatiza en los rasgos del sexo anatómico y explica la brecha por su impacto en las diferencias de género. Esta tipología no obedece a la especialización de los autores ni al uso de marcos teóricos fijos, sino al énfasis puesto sobre determinadas instancias de análisis: individuo, cultura o sexo anatómico.

\subsection{Explicación psicológica: énfasis en el individuo}

Al centrarse en el individuo, las explicaciones psicológicas se ocupan de la toma de decisio- nes. Aunque la brecha STEM sea un problema principalmente sociológico, la dimensión individual no debe descuidarse. Según Saucerman y Vasquez (2014), de la infancia a la adultez, las mujeres se topan con diversos «mensajes» como, por ejemplo, que su fracaso en carreras STEM ocurre por falta de habilidad, que los hombres son «naturalmente» más talentosos para tareas científicas o que ser «femenina» se opone a ser académica. Tales códigos constituyen «barreras psicológicas» que alejan a las mujeres de las carreras STEM, por lo que «comprender estos disuasivos es un primer paso importante para hallar intervenciones efectivas que aumenten la participación femenina en la adultez» (p. 47). Para analizar tal situación, las explicaciones psicológicas refieren a términos como autoconcepto, autoconfianza, autoeficacia, creencias, percepciones, intereses y preferencias.

\section{a. Autoconcepto, autoconfianza y autoeficacia}

Mientras autoconcepto remite a la valoración que un individuo tiene de sí mismo, autoconfianza refiere a su propia convicción sobre determinada habilidad y autoeficacia alude a su real cumplimiento. En la brecha STEM, tales conceptos explican que quienes afirmen ser más capaces en determinadas actividades académicas tenderán a elegir carreras STEM. Según Wang, Eccles y Kenny (2013), que las mujeres no elijan carreras STEM no ocurre por su carencia de habilidad verbal o matemática, sino por la mayor probabilidad de que mujeres con alta habilidad matemática también posean alta habilidad verbal, lo cual hace posible que puedan elegir más carreras respecto los hombres con alta habilidad matemática y habilidad verbal moderada. En estos casos, el autoconcepto sobre las habilidades matemática y verbal juega un rol crucial. 
Si hay estudiantes con mayor autoconfianza en rubros científicas o tienen padres vinculados a dichas áreas, aquellos tendrán mayor probabilidad de elegir carreras STEM; asimismo, aunque sea poco probable que las mujeres elijan tales carreras, las estudiantes que mostraban niveles semejantes de autoconfianza tenían la misma probabilidad que los estudiantes de elegir carreras STEM (Moakler y Kim, 2014). Según Carrasco y Sánchez (2016), los principales factores que influyen en las decisiones de mujeres de estudiar matemáticas son el reconocimiento de la autoeficacia, el gusto por la materia y el influjo docente. Dicha evidencia «ejemplifica cómo lo que creemos que somos y podemos hacer, es decir, nuestra identidad, se define de manera colectiva, con base en nuestras interacciones con las demás personas y las percepciones que estas tengan de nosotros» (p. 135).

Para Tellhed, Bäckström y Björklund (2017), la subrepresentación femenina en carreras STEM se relaciona fuertemente con la baja autoeficacia hacia tales campos y con las bajas expectativas de «pertenencia social» a dichos ámbitos. Tales expectativas también explicaron el menor interés masculino por las carreras de salud, educación básica y esfera doméstica o HEED (Health care, Elementary Education, and the Domestic spheres). Según los autores, las intervenciones que buscan disminuir las brechas deben centrarse en la pertenencia social de las mujeres: para aumentar el interés femenino por las carreras STEM, se debe contrarrestar las creencias de competencia estereotipada de género para reafirmarles a las mujeres que son capaces de desarrollarse profesionalmente en dichos ámbitos. Asimismo, Green y Sanderson (2018) analizaron la persistencia y logro en educación superior en materias STEM, concluyendo que habilidad y autoeficacia impactaban consistentemente en el desempeño en tales materias.

\section{b. Creencias y percepciones}

Aunque los conceptos autoconfianza y autoeficacia remitan a creencias individuales sobre la propia habilidad, aquellas se sustentan en desempeños positivos. En cambio, los estudios que explican la brecha STEM por acción de creencias y percepciones no requieren que estas sean empíricamente sustentadas. Eccles y Wang (2016) examinaron las creencias motivacionales que impulsaron a estudiantes a elegir carreras STEM y concluyeron que las mujeres tenían menos probabilidad de poseerlas. Aquellas que sí, tenían mayor probabilidad de seleccionar carreras como biología o medicina, en lugar de matemática, física, ingeniería o computación. Según los autores, las creencias motivacionales predicen las elecciones laborales «mucho más allá de los aportes de la habilidad matemática y verbal, la matrícula en cursos de matemática de la escuela secundaria y la educación e ingresos familiares» (p. 104).

Tras explorar las percepciones de hombres y mujeres sobre las carreras STEM en distintas etapas (educación secundaria, superior y vida laboral), Sáinz (2017) sostuvo que, generalmente, «existe la creencia de que las mujeres son más capaces de desarrollar habilidades vinculadas a la lectura o los idiomas, habilidades estas que se han entendido como congruentes con el rol de género femenino» (p. 11). Asimismo, «existe la creencia ampliamente compartida de que los chicos son mejores que las chicas en asignaturas que se consideran congruentes con el rol de género masculino, como las matemáticas, la física, la tecnología o el mundo científico en general» (Ibíd., pp. 11-12). Para la autora, la subrepresentación femenina en carreras STEM «se explica en parte por la imagen negativa que la gente joven tiene de las actividades que se desarrollan en muchos de estos ámbitos» (Ibíd., 14) y de sus realizadores, quienes son conside- 
rados «frikis» con «pocas habilidades sociales, pero, en contrapartida, son muy inteligentes» (Ibíd., p. 103).

No solo las creencias de las electoras influyen su elección de carrera. Las creencias de profesores sobre la «fijeza» o «maleabilidad» de las capacidades de los estudiantes afectan su motivación y logro académico al fungir de «barreras»: los profesores de «mentalidad fija» son más propensos a juzgar la inteligencia y la habilidad como capacidades «innatas», ocasionando que aquellos estudiantes pertenecientes a minorías étnicas experimenten menor motivación y bajo desempeño en relación a sus pares no juzgados (Canning, Muenks, Green y Murphy, 2019). Estos hallazgos indican que las «creencias sobre la mentalidad de la facultad» predicen las experiencias de los estudiantes en materias STEM, así como la magnitud de las brechas de rendimiento racial. Asimismo, las brechas de logros raciales en materias impartidas por facultades con mentalidad más «fija» fueron dos veces mayores que las dictadas por facultades con mentalidad «de mayor crecimiento». En conclusión, las creencias sobre la mentalidad de la facultad constituyen importantes predictores del logro y la motivación estudiantil en carreras STEM, más allá de otras características como género, etnia, edad, experiencia o jerarquía docente.

\section{c) Diferencias en intereses y preferencias}

Para Hakim (2006) las brechas laborales de género son amplias y algunas carreras son imposibles de ser «domesticadas» en pro de la igualdad de oportunidades. Según la autora, «hay evidencia sólida de que hombres y mujeres continúan siendo diferentes, en promedio, en sus orientaciones laborales y en su comportamiento en el mercado laboral» (p. 280), por lo que es «poco realista» esperar que la mujer ocupe la mitad de los puestos de trabajo. Al respecto, la «única teoría» que explica aquellas tendencias es la teoría de la preferencia, la cual «predice una polarización de los estilos de vida laboral como resultado de la diversidad en las preferencias de roles sexuales de las mujeres» (p. 286). Dicha teoría podría explicar por qué las diferencias de interés entre hombres y mujeres son amplias y persistentes en sociedades con mayor equidad de género (Lippa, 2010). Para Ceci y Williams (2015), las preferencias constituyen uno de los factores que mejor explican la brecha STEM.

Según Lippa, Preston y Penner (2014) las mujeres exponen fuertes intereses por trabajos orientados hacia las personas (people-oriented jobs) en contextos de menor igualdad, al ser «más libres» de «expresar sus intereses y valores mediante sus elecciones ocupacionales» (p. 7). Caso contrario, en países subdesarrollados, las mujeres no tienen el «lujo» de buscar un «trabajo basado en sus intereses», pues deben aceptar cualquier ocupación disponible (Ibíd.). Asimismo, para Su y Rounds (2015), mientras los hombres prefieren carreras orientadas hacia las cosas (things-orientation), las mujeres prefieren las orientadas hacia las personas (people-orientation). Según los autores, si buscamos comprender la subrepresentación femenina en carreras STEM, es necesario incidir en los intereses, pues tanto la elección entre carreras STEM y no STEM (como la ocurrida entre diversas carreras STEM) reflejan «patrones de interés individual».

\subsection{Explicación sociocultural: énfasis en la cultura}

Dado que la elección de carrera se realiza en marcos culturales, las explicaciones socioculturales sobre la brecha STEM enfatizan en la cultura, apelando a conceptos como influencia parental y socialización, discriminación y sesgos, y este- 
reotipos y roles de género. Según Schein (1984), la cultura funciona como un «entorno» para las carreras, por lo que su estudio se encuentra «inevitablemente ligado» a ella. Actualmente, diversos análisis confirman que la cultura es un aspecto clave de la elección de carrera (Özbilgin y MalachPines, 2007; Thege, Popescu-Willigmann, Pioch y Badri-Hoher, 2014), ya que dicho proceso «se hace en el contexto de una realidad social compleja que presenta a cada individuo una amplia variedad de opciones» (Eccles y Wang, 2016, p. 100). La cultura cumple un «rol» en tanto constituye el entorno de las elecciones: en culturas colectivistas la influencia parental ejerce mucha influencia, mientras en las individualistas los intereses personales tienen mayor significatividad (Akosah-Twumasi et al., 2018).

En carreras científicas, la cultura importa: diversos factores ambientales (estereotipos, cultura o roles) contribuyen a la brecha STEM y afectan a las mujeres en diversas instancias: educación básica, secundaria, superior y mercado laboral (Kahn y Ginther, 2017). Tras revisar estudios realizados en los últimos 30 años, Wang y Degol (2017) sostuvieron que la brecha STEM constituye un «fenómeno cultural» provocado por la «compleja interacción» de seis factores: diferencias en habilidad cognitiva, fortalezas cognitivas relativas, preferencias laborales, preferencias en estilo de vida, creencias sobre habilidades específicas, y estereotipos de género y sesgos. Asimismo, resaltando la influencia del entorno, Lippmann y Senik (2018) demostraron que el bajo rendimiento matemático infantil se redujo drásticamente en la antigua Alemania democrática, en comparación con la otrora Alemania federal. Para los autores, la diferencia entre Oriente y Occidente se debe a las actitudes, confianza y competitividad de las niñas en matemáticas, y no a las diferencias económicas.

\section{a. Influencia parental y socialización.}

Una primera forma de comprender el influjo cultural en la brecha STEM es reconociendo el peso de la influencia parental y la socialización. Buschor, Berweger, Frei y Kappler (2014) analizaron durante 2 años si mujeres estudiantes de secundario elegían carreras STEM y cómo percibieron dicho proceso retrospectivamente, y hallaron que existe una «alta persistencia» a estudiar carreras STEM, la cual decrece en la transición hacia la educación superior. El análisis cualitativo realizado reveló que las experiencias de aprendizaje, el apoyo parental y los modelos a seguir fueron decisivos en la elección de carrera de las estudiantes. De modo semejante, para Eccles (2014) la influencia familiar sobre las carreras STEM se ejerce a través de la socialización de género. Enfocándose en dicho proceso, la autora analizó cómo las percepciones de los padres sobre las habilidades académicas de sus hijos predicen la confianza que ellos tendrán sobre sus propias habilidades, cómo tales diferencias se trasmiten según el género de los hijos y cómo tales predicen sus percepciones y conductas.

\section{b. Discriminación y sesgos}

Diversos estudios afirman que la brecha STEM ocurre porque las mujeres son discriminadas en el acceso a ciertas carreras científicas. La literatura al respecto es amplia, especialmente entre poblaciones nativo americanas, afroamericanas y latinas (Perna et al., 2009; Johnson, 2011; Syed y Chemers, 2011; Jackson, Starobin y Laanan, 2013; Mack, Rankins y Woodson, 2013; Canning, Muenks, Green y Murphy, 2019). La poca contratación femenina en carreras STEM es clave para entender la brecha (McNeely y Vlaicu, 2010). Las mujeres no solo son menos contratadas al ser vistas como menos competentes (Moss-Racusin, Dovidio, Brescoll, Graham y Handelsman, 2012), 
sino que también hay sesgos que favorecen a los varones, pues estos evalúan las investigaciones sobre sesgos de género de forma menos favorable que las mujeres (Handley, Brown, Moss-Racusin y Smith, 2015). Según Farrell y McHugh (2017), las personas que estudian o trabajan en carreras STEM exhiben diferentes niveles de sesgo implícito de hombres-STEM que aquellos que trabajan en carreras no STEM. Sin embargo, también existen sesgos pro mujeres-STEM entre mujeres.

Ceci, Ginther, Kahn y Williams (2014) compararon extensivamente (desde la escuela secundaria hasta la educación superior y la etapa laboral) las trayectorias de mujeres en carreras STEM y noSTEM, y hallaron que la discriminación de género es una hipótesis actualmente no válida. Si bien la mayoría de estudios apunta a la discriminación o al sexismo como factor principal de la brecha, la evidencia reciente considera que muchas de sus afirmaciones no son válidas (Ceci y Williams, 2011). Según Jussim (2017), no solo existen otras explicaciones mejores (diferencias en intereses o habilidades cognitivas), sino también campos STEM y no STEM donde las mujeres se hallan sobrerrepresentadas. Tras analizar las tendencias de contratación de científicas, Williams y Ceci (2015) concluyeron que, en realidad, estamos en un momento propicio para que las mujeres inicien carreras académicas. Aparte de los sesgos contra las mujeres en carreras STEM y contra algunos estudios que aplican solo participantes hombres, existe un tercer sesgo: el de la investigación sobre el sesgo de género. Cislak, Formanowicz y Saguy (2018) realizaron un análisis bibliométrico y demostraron que los estudios sobre el tema son menos financiados y publicados.

\section{c. Estereotipos y roles de género}

Explicar la brecha STEM mediante estereotipos y roles de género constituye la forma más común desde la perspectiva sociocultural. Los estereotipos son «creencias, prescripciones, reglas, expectativas y atribuciones la mayoría de veces implícitas sobre cómo hombres y mujeres deben comportarse, es decir, en los estereotipos de género se define lo que en cada sociedad se entiende como masculino o femenino» (Verde et al., 2007, p. 47). En relación a la brecha STEM, los estereotipos constituyen un factor que expone la importancia del contexto cultural (Nassar-McMillan, Wyer, Oliver-Hoyo y Schneider, 2011; Prives, 2013). Cheryan, Davies, Plaut y Steele (2009) estudiaron los estereotipos sobre la computación (carrera mayormente poblada por hombres) y tras modificarlos lograron generar interés femenino por dicha carrera. Dependiendo de cuánto algunas áreas vinculadas a las matemáticas estén estereotipadas como «masculinas», se podría explicar por qué las mujeres no eligen tales carreras (Cheryan, 2012). La literatura al respecto es amplia.

Smeding (2012) estudió los estereotipos de género en carreras STEM y su relación con el desempeño en estudiantes de ingeniería y humanidades, y halló que las estudiantes de ingeniería tenían estereotipos de género más débiles que las estudiantes de humanidades y los estudiantes de ingeniería y humanidades. Ramsey, Betz y Sekaquaptewa (2013) compararon las mujeres en campos STEM de entornos acogedores con las de entornos tradicionales, y demostraron la importancia de un ambiente acogedor para las mujeres en dichas carreras. Asimismo, Jackson, Hillard y Schneider (2014) hallaron que los hombres eran más propensos que las mujeres a respaldar los estereotipos sobre mujeres en carreras STEM, razón por la cual concluyeron que los estereotipos afectan negativamente la educación, contratación, promoción y retención de mujeres en tales carreras. Según Cheryan, Master y Meltzoff (2015), los 
«estereotipos académicos» de ciertas carreras sirven como «guardianes» que alejan a las mujeres de ciertos campos. Por ejemplo, computación e ingeniería son carreras estereotipadas como orientadas hacia varones porque implica aislamiento social y enfoque en máquinas.

Para Master y Meltzoff (2017) los estereotipos culturales que asocian las carreras STEM a los varones actúan como «barreras» que impiden que las mujeres se interesan por tales profesiones. Estos estereotipos son adoptados en la infancia y pueden influenciar las actitudes académicas y el desempeño de las mujeres. No obstante, está demostrado que modificarlos genera un mayor interés femenino por las carreras STEM. Según Cheryan, Ziegler, Montoya y Jiang (2017), hay 3 factores generales que explican la brecha STEM: culturas masculinas en carreras STEM que alejan a las mujeres por no sentir pertenencia, falta de experiencia en dichas carreras y brechas en autoeficacia. Asimismo, los procesos motivacionales están influenciados por los roles sociales: las carreras STEM no son percibidas como aquellas capaces de brindar oportunidades para ayudar al prójimo; comprender estas percepciones contribuye al conocimiento sobre las diferencias entre tales carreras y otras vías profesionales, así como las elecciones de hombres y mujeres (Diekman, Steinberg, Brown, Belanger y Clark, 2017).

Drury, Siy y Cheryan (2011) sostuvieron que, aunque los modelos femeninos puedan ser efectivos para la retención de mujeres en carreras STEM, los modelos masculinos y femeninos son igualmente efectivos para el reclutamiento. Olsson y Martiny (2018) demostraron que es posible promover el ingreso de mujeres a campos donde están subrepresentadas y estereotipadas negativamente (por ejemplo, carreras STEM) mediante el empleo de modelos contraestereotípicos. Según Starr (2018), el estereotipo de «nerd genio»y los estereotipos de género contribuyen negativamente a la identificación de las mujeres con las carreras STEM y a su motivación a elegir tales carreras. Makarova, Aeschlimann y Herzog (2019) analizaron el impacto de la imagen de masculinidad de tres materias (química, matemática y física) en estudiantes de secundaria, hallando que la matemática era considerada la carrera con la masculinidad más fuerte, seguida por la física y la química. Al demostrar que una imagen masculina menos pronunciada aumenta la probabilidad de elegir carreras STEM, los autores concluyeron que los estereotipos sobre las profesiones y el género influyen en las decisiones de hombres y mujeres.

Los estudios sobre estereotipos de género y brecha STEM son tantos que han postulado la teoría de amenaza de estereotipo (stereotype threat theory), la cual explica cómo los estereotipos influyen de manera negativa en el desempeño profesional de las mujeres (Shapiro y Williams, 2012; Shaffer, Marx y Prislin, 2013; Casad, Hale y Wachs, 2017; Bedyńska, Krejtz y Sedek, 2018; Canning et al., 2019). Tras una revisión de estudios que refieren a dicha teoría, Régner, Steele, Ambady, Thinus-Blanc y Huguet (2014) definieron la amenaza de estereotipo como la afectación que sufren las mujeres en su rendimiento matemático al ser estereotipadas negativamente. Para los autores, dichas amenazas empezarían a formarse muy temprano en la escuela primaria hasta consolidarse, a lo largo del tiempo, como barreras «ocultas» y opuestas al avance futuro de las mujeres ya en ámbitos laborales científicos. En otras palabras, los estereotipos negativos son capaces de afectar prácticamente toda la vida educativa y laboral de las mujeres.

\subsection{Explicación biológica}

Inusuales, pero no ausentes, hay estudios que brindan una explicación biológica de la brecha 
STEM. Tales investigaciones, aunque no nieguen la existencia de diversos factores psicológicos y socioculturales, enfatizan en cómo los rasgos del sexo anatómico entre hombres y mujeres influyen en la elección de carrera. Apoyándose en cuantiosa evidencia transcultural que indica que las diferencias de género (en temperamento, desempeño cognitivo y conducta) son constantes, la explicación biológica sostiene que las brechas en intereses y preferencias también estarían fuertemente influenciadas por las diferencias sexuales. Para Browne (2005), la «historia completa» no podrá ser comprendida si no consideramos las diferencias sexuales, ya que «el rol de la biología no se limita a las diferencias en fuerza física; hombres y mujeres difieren (en promedio) en rasgos cognitivos y temperamentales que afectan su talento e intereses en ocupaciones particulares» (p. 510). Para el autor, que las diferencias psicológicas entre hombres y mujeres se incrementen en la pubertad no es evidencia de la fuerza de la socialización, sino resultado de «tumultuosos cambios hormonales». En la misma tónica, Sapienza, Zingales y Maestripieri (2009) afirmaron que los niveles de testosterona prenatal y la mayor aversión al riesgo pueden predecir la elección de carrera: individuos con mayores niveles de testosterona y baja aversión al riesgo (hombres) tenían mayor probabilidad de elegir carreras que implican riesgos (como las finanzas).

El estudio realizado por Lippa (2010) también puede ser incluido en esta tipología al proponer que, dado que las diferencias de género son consistentes en diversas culturas y épocas, podrían deberse a factores biológicos. Para los defensores de la tesis biológica, existe amplia evidencia de cómo ciertos rasgos biológicos afectan la conducta, posibilitando, entre otras cosas, que las mujeres se interesen por ocupaciones orientadas a las personas (people-oriented), mientras los hombres prefieran actividades orientadas a las cosas (thing-oriented) ( $\mathrm{Su}$, Rounds y Armstrong, 2009; Yang y Barth, 2015). Nater-Otero (2017) estudió los «factores evolutivos» que contribuyen a la brecha STEM (inversión de descendencia, mayor inversión en descendencia masculina y aversión al riesgo femenino) para explicar por qué las carreras STEM no son pensadas como un lugar para las mujeres, por qué el género influye en las políticas académicas y laborales, y cómo esto ha conllevado a discrepancias sobre la brecha STEM. Asimismo, el estudio realizado por O'Dea, Lagisz, Jennions y Nakagawa (2018) puede ser incluido en esta tipología, al estar basado en la denominada hipótesis de la variabilidad masculina.

\section{Conclusiones}

El presente ensayo realiza una revisión de literatura sobre la brecha STEM y la organiza según sus tres tipos de explicación: psicológica, sociocultural y biológica. Dicha tipología permite comprender no solo los marcos teóricos de los estudios realizados, sino también sus instancias centrales de análisis, conceptos eje y principales hallazgos. Aunque tales explicaciones compongan las tres dimensiones de un mismo problema, el debate sigue abierto: explicaciones distintas no significa explicaciones igualmente válidas. En lo que a ciencia refiere, siempre habrá argumentos mejores que otros. Tras revisar más de 400 estudios, Ceci, Williams y Barnett (2009) afirmaron que la evidencia de discriminación es anticuada y anecdótica, mientras las hipótesis biológicas no demuestran causalidad: «los datos no son lo suficientemente consistentes como para afirmar que la escasez de mujeres en carreras STEM se haya demostrado principalmente como consecuencia directa de diferencias sexuales biológicas (por ejemplo, genes, hormonas) [...] Hay demasiada inconsistencia entre estudios, 
tests, culturas y, ocasionalmente, entre épocas para justificar un fuerte papel directo del sexo biológico» (p. 249).

Desde luego, los factores señalados en este trabajo no son los únicos que influencian la brecha STEM. Las diferencias en formación y experiencias educativas explican gran parte de tales diferencias (Griffith, 2010). Dasgupta y Stout (2014) hallaron que las causas de la brecha STEM dependen de 3 etapas del desarrollo (niñez y adolescencia, inicio de la adultez y adultez) y que ciertos entornos de aprendizaje, relaciones entre pares y características familiares pueden ser obstáculos para las mujeres. Asimismo, no toda la literatura sobre la brecha STEM busca explicar su origen. Dunlap, Barth y Chappetta (2019) estudiaron los valores familiares de mujeres tanto en carreras dominadas por hombres (STEM) y dominadas por mujeres, y concluyeron que las parejas en carreras STEM mostraban diferencias de género limitadas y opuestas a los estereotipos. Caso contrario, las parejas en carreras dominadas por mujeres exponían la imagen tradicional de esposo proveedor y mujer criadora.

Para Wang y Degol (2017), que los factores socioculturales influyan fuertemente en la elección de carrera significa que es posible intervenir tales resultados. Al respecto, diversos estudios han desarrollado programas que contribuyen al acceso de mujeres a carreras STEM con resultados positivos (Inkelas, 2011; Szelényi y Inkelas, 2011; Szelényi, Denson y Inkelas, 2013; Botella, Rueda, LópezIñesta y Marzal, 2019). Considerando el éxito y bienestar de las mujeres en carreras STEM han sido referidos (Settles, 2014), la preocupación por dicha problemática generará no solo desarrollo social, sino también desarrollo científico. En palabras de Arredondo, Vázquez y Velázquez (2019), «la igualdad de género en campos de STEM debe ser vista, no únicamente como una cuestión [...] de derechos humanos básicos, sino como un medio fundamental para promover la excelencia científica y tecnológica» (p. 154). Que el presente trabajo sea un aporte a dicho fin. 


\section{Bibliografía}

Akosah-Twumasi, P., Emeto, T., Lindsay, D., Tsey, K. \& Malau-Aduli, B. (2018). A systematic review of factors that influence youths career choices-the role of culture. Frontiers in Education, 3, art. 58.

Amarasekara, I. \& Grant, W. (2019). Exploring the YouTube science communication gender gap: A sentiment analysis. Public Understanding of Science, 28(1), 68-84.

Arredondo, F, Vázquez, J. \& Velázquez, L. (2019). STEM y brecha de género en Latinoamérica. Revista de El Colegio de San Luis, 9(18), 137-158.

Bedyńska, S., Krejtz, I. \& Sedek, G. (2018). Chronic stereotype threat is associated with mathematical achievement on representative sample of secondary schoolgirls: The role of gender identification, working memory, and intellectual helplessness. Frontiers in Psychology, 9, art.428.

Botella, C., Rueda, S., López-Iñesta, E. \& Marzal, P. (2019). Gender diversity in STEM disciplines: A multiple factor problem. Entropy, 21(1), 1-17.

Brown, D. (2002). Introduction to theories of career development and Choice: Origins, evolution, and current efforts, en Career choice and development (pp. 3-23). San Francisco: Wiley.

Browne, K. (2005). Women in science: Biological factors should not be ignored. Cardozo Women's Law Journal, 11(3), 509-528.

Buschor, C., Berweger, S., Frei, A. \& Kappler, C. (2014). Majoring in STEM-What accounts for women's career decision making? A mixed methods study. The Journal of Educational Research, 107(3), 167-176.

Bystydzienski, J. \& Bird, S. (2006). Removing barriers: Women in academic science, technology, engineering, and mathematics. United States of America: Indiana University Press. Canning, E., Muenks, K., Green, D. \& Murphy, M. (2019). STEM faculty who believe ability is fixed have larger racial achievement gaps and inspire less student motivation in their classes. Science Advances, 5(2), eaau4734.

Carrasco, L. \& Sánchez, M. (2016). Factores que favorecen la elección de las matemáticas como profesión entre mujeres estudiantes de la Universidad Veracruzana. Perfiles Educativos, 38(151), 123-138.

Casad, B., Hale, P. \& Wachs, F. (2017). Stereotype threat among girls: Differences by gender identity and math edu- cation context. Psychology of Women Quarterly, 41(4), 513529.

Ceci, S. \& Williams, W. (2011). Understanding current causes of women's underrepresentation in science. Proceedings of the National Academy of Sciences, 108(8), 3157-3162.

Ceci, S. \& Williams, W. (2015). Why so few women in mathematically intensive fields? En R. Scott y S. Kosslyn (eds.), Emerging trends in the social and behavioral sciences (pp. 1-12), US: Wiley.

Ceci, S., Williams, W. \& Barnett, S. (2009). Women's underrepresentation in science: Sociocultural and biological considerations. Psychological Bulletin, 135(2), 218-261.

Ceci, S., Ginther, D., Kahn, S. \& Williams, W. (2014). Women in academic science: A changing landscape. Psychological Science in the Public Interest, 15(3), 75-141.

Cheryan, S. (2012). Understanding the paradox in mathrelated fields: Why do some gender gaps remain while others do not? Sex Roles, 66(3-4), 184-190.

Cheryan, S., Davies, P., Plaut, V. \& Steele, C. (2009). Ambient belonging: How stereotypical cues impact gender participation in computer science. Journal of Personality and Social Psychology, 97(6), 1045-1060.

Cheryan, S., Master, A. \& Meltzoff, A. (2015). Cultural stereotypes as gatekeepers: Increasing girls' interest in computer science and engineering by diversifying stereotypes. Frontiers in Psychology, 6, art.49.

Cheryan, S., Ziegler, S., Montoya, A. \& Jiang, L. (2017). Why are some STEM fields more gender balanced than others? Psychological Bulletin, 143(1), 1-35.

Cislak, A., Formanowicz, M. \& Saguy, T. (2018). Bias against research on gender bias. Scientometrics, 115, 189-200.

Colaner, C. \& Giles, S. (2008). The baby blanket or the briefcase: The impact of evangelical gender role ideologies on career and mothering aspirations of female evangelical college students. Sex Roles, 58(7-8), 526-534.

Correll, S. (2004). Constraints into preferences: Gender, status, and emerging career aspirations. American Sociological Review, 69(1), 93-113.

Dasgupta, N. \& Stout, J. (2014). Girls and women in science, technology, engineering, and mathematics: STEMing the tide and broadening participation in STEM careers. Policy Insights from the Behavioral and Brain Sciences, 1(1), 21-29.

Diekman, A., Steinberg, M., Brown, E., Belanger, A. \& Clark, E. (2017). A goal congruity model of role entry, en- 
gagement, and exit: Understanding communal goal processes in STEM gender gaps. Personality and Social Psychology Review, 21(2), 142-175.

Drury, B., Siy, J. \& Cheryan, S. (2011). When do female role models benefit women? The importance of differentiating recruitment from retention in STEM. Psychological Inquiry, 22(4), 265-269.

Dunlap, S., Barth, J. \& Chappetta, K. (2019). Gender roles in the romantic relationships of women in STEM and female-dominated majors: A study of heterosexual couples. Gender Issues, 36(2), 113-135.

Eccles, J. (2014). Gendered socialization of STEM interests in the family. 2nd Network Gender \& STEM Conference. Berlin, Germany.

Eccles, J. \& Wang, M. (2016). What motivates females and males to pursue careers in mathematics and science? International Journal of Behavioral Development, 40(2), 100-106.

Farrell, L. \& McHugh, L. (2017). Examining gender-STEM bias among STEM and non-STEM students using the Implicit Relational Assessment Procedure (IRAP). Journal of Contextual Behavioral Science, 6(1), 80-90.

Fausto-Sterling, A. (1981). Women and science. Women's Studies International Quarterly, 4(1), 41-50.

Feeney, M. \& Bernal, M. (2010). Women in STEM networks: Who seeks advice and support from women scientists? Scientometrics, 85, 767-790.

Green, A. \& Sanderson, D. (2018). The roots of STEM achievement: An analysis of persistence and attainment in STEM majors. The American Economist, 63(1), 79-93.

Griffith, A. (2010). Persistence of women and minorities in STEM field majors: Is it the school that matters? Economics of Education Review, 29, 911-922.

Hakim, C. (2006). Women, careers, and work-life preferences. British Journal of Guidance E Counselling, 34(3), 279-294.

Handley, I., Brown, E., Moss-Racusin, C. \& Smith, J. (2015). Quality of evidence revealing subtle gender biases in science is in the eye of the beholder. Proceedings of the National Academy of Sciences, 112(43), 13201-13206.

Inkelas, K. (2011). Living-learning programs for women in STEM. New Directions for Institutional Research, 152, 27-37. Jackson, D., Starobin, S. \& Laanan, F. (2013). The shared experiences: Facilitating successful transfer of women and underrepresented minorities in STEM fields. New Directions for Higher Education, 162, 69-76.
Jackson, S., Hillard, A. \& Schneider, T. (2014). Using implicit bias training to improve attitudes toward women in STEM. Social Psychology of Education, 17, 419-438.

Jiang, S., Schenke, K., Eccles, J., Xu, D. \& Warschauer, M. (2018). Cross-national comparison of gender differences in the enrollment in and completion of science, technology, engineering, and mathematics Massive Open Online Courses. PLoS ONE, 13(9), e0202463.

Johnson, D. (2011). Women of color in science, technology, engineering, and mathematics (STEM). New Directions for Institutional Research, 152, 75-85.

Jussim, L. (2017). Why brilliant girls tend to favor nonSTEM careers. Psychology Today. Disponible en: https:// www.psychologytoday.com/us/blog/rabble-rouser/201707/ why-brilliant-girls-tend-favor-non-stem-careers

Kahn, S. \& Ginther, D. (2017). Women and STEM. Working paper 23525. Cambridge: National Bureau of Economic Research.

Kazi, A. \& Akhlaq, A. (2017). Factors affecting students' career choice. Journal of Research and Reflections in Education 2, 187-196

Kwon, E. (2017). «For passion or for future family?» Exploring factors influencing career and family choices of female medical students and residents. Gender Issues, 34, 186-200. Lightbody, P., Siann, G., Tait, L. \& Walsh, D. (1997). A fulfilling career? Factors which influence women's choice of profession. Educational Studies, 23(1), 25-37.

Lippa, R. (2010). Gender differences in personality and interests: When, where, and why? Social Personality Psychology Compass, 4(11), 1098-1110.

Lippa, R., Preston, K. \& Penner, J. (2014). Women's representation in 60 occupations from 1972 to 2010: More women in high-status jobs, few women in things-oriented jobs. PLoS ONE, 9(5), e95960.

Lippmann, Q. \& Senik, C. (2018). Math, girls and socialism. Journal of Comparative Economics, 46(3), 874-888.

López-Bassols, V., Grazzi, M., Guillard, C. \& Salazar, M. (2018). Las brechas de género en ciencia, tecnología e innovación en América Latina y el Caribe. Banco Interamericano de Desarrollo.

Mack, K., Rankins, C. \& Woodson, K. (2013). From graduate school to the STEM workforce: An entropic approach to career identity development for STEM women of color New Directions for Higher Education, 136, 23-34. 
Makarova, E., Aeschlimann, B. \& Herzog, W. (2019). The gender gap in STEM fields: The impact of the gender stereotype of math and science on secondary students' career aspirations. Frontiers in Education, 4, art. 60.

Malik, S. \& Al-Emran, M. (2018). Social factors influence on career choices for female computer science students. International Journal of Emerging Technologies in Learning, 13(5), 56-70.

Master, A. \& Meltzoff, A. (2017). Building bridges between psychological science and education: Cultural stereotypes, STEM, and equity. Prospects, 46(2), 215-234.

McNeely, C. \& Vlaicu, S. (2010). Exploring institutional hiring trends of women in the U.S. STEM professoriate. Review of Policy Research, 27(6), 781-793.

Moakler, M. \& Kim, M. (2014). College major choice in STEM: Revisiting confidence and demographic factors. The Career Development Quarterly, 62(2), 128-142.

Moss-Racusin, C., Dovidio, J., Brescoll, V., Graham, M. \& Handelsman, J. (2012). Science faculty's subtle gender biases favor. Proceedings of the National Academy of Sciences, 109(41), 16474-16479.

Mosteiro, M. (1997). El género como factor condicionante de la elección de carrera: Hacia una orientación para la igualdad de oportunidades entre los sexos. Revista GalegoPortuguesa de Psicoloxía e Educación, 1, 305-315.

Nassar-McMillan, S., Wyer, M., Oliver-Hoyo., M. \& Schneider, J. (2011). New tools for examining undergraduate students' STEM stereotypes: Implications for women and other underrepresented groups. New Directions for Institutional Research, 152, 87-98.

Nater-Otero, Y. (2017). Biological factors in the STEM gender gap [Tesis de maestría]. Montclair: Montclair State University. O’Dea, R., Lagisz, M., Jennions, M. \& Nakagawa, S. (2018). Gender differences in individual variation in academic grades fail to fit expected patterns for STEM. Nature Communications, 9, 3777.

Olsson, M. \& Martiny, S. (2018). Does exposure to counterstereotypical role models influence girls' and women's gender stereotypes and career choices? A review of social psychological research. Frontiers in Psychology, 9, 2264.

Özbilgin, M. \& Malach-Pines, A. (Eds.). (2007). Career choice in management and entrepreneurship: A research companion. United Kingdom: Edward Elgar.
Perera, J. \& Velummayilum, P. (2008). Women's career choice and gender roles: A South Asian experience. The Clinical Teacher, 5, 186-190.

Perna, L., Lundy-Wagner, V., Drezner, N., Gasman, M., Yoon, S., Bose, E. \& Gary, S. (2009). The contribution of HBCUS to the preparation of African American women for stem careers: A case study. Research in Higher Education, 50(1), 1-23.

Prives, L. (2013). Studying stereotypes: Examining why many women do not pursue STEM careers. IEEE Women in Engineering Magazine, 7(2), 15-17.

Ramsey, L., Betz, D. \& Sekaquaptewa, D. (2013). The effects of an academic environment intervention on science identification among women in STEM. Social Psychology of Education, 16(3), 377-397.

Régner, I., Steele, J., Ambady, N., Thinus-Blanc, C. \& Huguet, P. (2014). Our future scientists: A review of stereotype threat in girls from early elementary school to middle school. Revue Internationale de Psychologie Sociale, 27(3-4), 13-51.

Reinking, A. \& Martin, B. (2018). The gender gap in STEM fields: Theories, movements, and ideas to engage girls in STEM. Journal of New Approaches in Educational Research, 7(2), 148-153.

Rodríguez-Muñiz, L., Areces, D., Suárez-Álvarez, J., Cueli, M. \& Muñiz, J. (2019). ¿Qué motivos tienen los estudiantes de Bachillerato para elegir una carrera universitaria? Revista de Psicología y Educación, 14(1), 1-15.

Ruiz-Gutiérrez, J. \& Santana-Vega, L. (2018). Elección de carrera y género. Revista Electrónica de Investigación y Docencia, 19, 7-20.

Sáinz, M. (Coord.). (2017). Se buscan ingenieras, fisicas y tecnólogas. ¿Por qué no hay más mujeres STEM? Barcelona: Ariel.

Sapienza, P., Zingales, L. y Maestripieri, D. (2009). Gender differences in financial risk aversion and career choices are affected by testosterone. Proceedings of the National Academy of Sciences, 106(36), 15268-15273.

Saucerman, J. \& Vasquez, K. (2014). Psychological barriers to STEM participation for women over the course of development. Adultspan Journal, 13(1), 46-64.

Schein, E. (1984). Culture as an environmental context for careers. Journal of Occupational Behaviour, 5, 71-81.

Settles, I. (2014). Women in STEM: Challenges and determinants of success and well-being. American Psychological 
Association. Disponible en: https://www.apa.org/science/ about/psa/2014/10/women-stem

Shahzad, M., Fatima, S. \& Ahmed, M. (2018). Factors influencing on students' career choices: Multivariate analysis. International Journal of Contemporary Applied Researches, 5(6), 1-16.

Shaffer, E., Marx, D. \& Prislin, R. (2013). Mind the gap: Framing of women's success and representation in STEM affects women's math performance under threat. Sex Roles, 68(7-8), 454-463.

Shapiro, C. \& Sax, L. (2011). Major selection and persistence for women in STEM. New Directions for Institutional Research, 152, 5-18.

Shapiro, J. \& Williams, A. (2012). The role of stereotype threats in undermining girls' and women's performance and interest in STEM fields. Sex Roles, 66(3-4), 175-183.

Sharif, N., Ahmed, N. \& Sarwar, S. (2019). Factors influencing career choices. IBT Journal of Business Studies, 15(1), 33-46.

Smeding, A. (2012). Women in Science, Technology, Engineering, and Mathematics (STEM): An investigation of their implicit gender stereotypes and stereotypes' connectedness to math performance. Sex Roles, 67(11-12), 617-629.

Smith, E. (2011). Women into science and engineering? Gendered participation in higher education STEM subjects. British Educational Research Journal, 37(6), 993-1014.

Starr, C. (2018). «I'm not a science nerd!»: STEM stereotypes, identity, and motivation among undergraduate women. Psychology of Women Quarterly, 42(4), 489-503.

Stoet, G. \& Geary, D. (2018). The gender-equality paradox in science, technology, engineering, and mathematics education. Psychological Science, 29(4), 581-593.

Su, R. \& Rounds, J. (2015). All STEM fields are not created equal: People and things interests explain gender disparities across STEM fields. Frontiers in Psychology, 6, 189.

Su, R., Rounds, J. \& Armstrong, P. (2009). Men and things, women and people: A meta-analysis of sex differences in interests. Psychological Bulletin, 135(6), 859-884.

Syed, M. \& Chemers, M. (2011). Ethnic minorities and women in STEM: Casting a wide net to address a persistent social problem. Journal of Social Issues, 67(3), 435-441.
Szelényi, K. \& Inkelas, K. (2011). The role of living-learning programs in women's plans to attend graduate school in STEM fields. Research in Higher Education, 52(4), 3494-369. Szelényi, K., Denson, N. \& Inkelas, K. (2013). Women in STEM majors and professional outcome expectations: The role of living-learning programs and other college environments. Research in Higher Education, 54(8), 851-873.

Tellhed, U., Bäckström, M. \& Björklund, F. (2017). Will I fit in and do well? The importance of social belongingness and self-efficacy for explaining gender differences in interest in STEM and HEED majors. Sex Roles, 77(1), 86-96.

Thege, B., Popescu-Willigmann, S., Pioch, R. \& BadriHoher, S. (Eds.). (2014). Paths to career and success for women in science. Wiesbaden: Springer.

Tien, H., Wang, Y. \& Liu, L. (2009). The role of career barriers in high school students' career choice behavior in Taiwan. The Career Development Quarterly, 57(3), 274-288. Verde, E., Gallardo, G., Compeán, S., Tamez, S. \& OrtizHernández, L. (2007). Motivos de elección de carrera en mujeres estudiantes de profesiones de la salud. Educación Médica, 10(1), 44-51.

Wang, M. \& Degol, J. (2017). Gender gap in science, technology, engineering, and mathematics (STEM): Current knowledge, implications for practice, policy, and future directions. Educational Psychology Review, 29(1), 119-140.

Wang, M., Eccles, J. \& Kenny, S. (2013). Not lack of ability but more choice: Individual and gender differences in choice of careers in science, technology, engineering, and mathematics. Psychological Science, 24(5), 770-775.

Williams, J. \& Massinger, K. (2016). How women are harassed out of science: The discrimination young researchers endure makes America's need for STEM workers even greater. The Atlantic. Disponible en: https:/www.theatlantic. com/science/archive/2016/07/how-women-are-harassedout-of-science/492521/

Williams, W. \& Ceci, S. (2015). National hiring experiments reveal 2:1 faculty preference for women on STEM tenure track. Proceedings of the National Academy of Sciences, 112(17), 5360-5365.

Yang, Y. \& Barth, J. (2015). Gender differences in STEM undergraduates' vocational interests: People-thing orientation and goal affordances. Journal of Vocational Behavior, 91, 65-75. 